\title{
Influence of a-lipoic acid on morphology of organs of rabbits fed a high fat diet with the addition of oxidised rapeseed oil
}

\author{
Barbara Stawiarska-Pięta ${ }^{1}$, Jolanta Zalejska-Fiolka², Magdalena Wyszyńska ${ }^{1}$, \\ Anna Kleczka ${ }^{1}$, Beata Janiga ${ }^{1}$, Natalia Grzegorzak ${ }^{1}$, Ewa Birkner ${ }^{2}$ \\ ${ }^{1}$ Department of Pathology, School of Pharmacy with the Division of Laboratory Medicine in Sosnowiec, \\ Medical University of Silesia in Katowice, 41-200 Sosnowiec, Poland \\ ${ }^{2}$ Department of General Biochemistry, School of Medicine with the Division of Dentistry in Zabrze, \\ Medical University of Silesia in Katowice, 41-808 Zabrze, Poland \\ bspieta@o2.pl
}

Received: May 25, $2017 \quad$ Accepted: November 20, 2017

\begin{abstract}
Introduction: The aim of the study was to assess the influence of $\alpha$-lipoic acid (ALA) on the morphology of the aorta and liver of rabbits fed high fat diet with addition of oxidised (ORO) and non-oxidised rapeseed oil (N-ORO). Material and Methods: The study was conducted on male chinchilla rabbits divided into six groups. The control group (C) was fed a breeding standard diet (BSD), group I received BSD with the addition of ALA in the dose of $10 \mathrm{mg} / \mathrm{kg}$ b.w., groups II and III received BSD enriched with $10 \%$ addition of $\mathrm{N}-\mathrm{ORO}$ or ORO, whereas rabbits from groups IV and V received BSD with $10 \%$ addition of N-ORO or ORO and ALA. Results: Addition of ORO caused necrosis and steatosis of hepatocytes, as well as atherosclerotic plaques of various intensification in the aorta. In the liver of rabbits from group II (N-ORO) infiltrations of mononuclear cells was observed in the area of liver triads and between liver lobules. The beneficial influence of ALA was demonstrated in rabbits fed a diet containing N-ORO or ORO. In case of ORO, the activity of ALA was not fully effective. Conclusion: Diet supplementation with ALA counteracts the changes generated in the liver and aorta under increased exposure to higher fat content in diet, in particular thermally treated fats.
\end{abstract}

Keywords: rabbits, liver, aorta, $\alpha$-lipoic acid, oxidised rapeseed oil.

\section{Introduction}

It has been demonstrated that high fat diets, particularly containing oxidised vegetable oils, are a significant risk factor for civilisation-related diseases, such as diseases of the cardio-vascular system $(19,26)$. Oxidation of fats included in many foodstuffs, occurring during storage or food treatment for culinary purposes, causes reduction of their biological and nutritive properties. The responsibility for noxious activity rests both with the increased amount of free radicals and reactive oxygen species, as well as the end products of lipid peroxidation, namely 4-hydroxynonenal, or malondialdehyde. They demonstrate a strong cytotoxic activity. It was demonstrated that after digestion part of them is absorbed to lymph or directly to blood. Consumption of oxidised fats increases their excretion in urine in humans and animals, in the form of malondialdehyde, but also in the form of lipophilic carbonyl compounds (1). Harmful products of lipid oxidation activate inflammatory response in the circulatory system, liver, intestines, and kidneys (22,23).

Oxidised lipids may be supplied with incorrectly stored or thermally treated food, but may also occur in the vessel walls, where they cumulate in the course of atherogenesis. They act unfavourably for cell metabolism and damage cell membranes leading to cell necrosis (2). Studies have demonstrated the atherogenic mechanism of oxidised LDL $(5,18)$.

Numerous studies have shown that both excess amount of fats and the presence of oxidised fats in the diet cause changes in the vessels in the form of atherosclerotic plaques. Particularly unfavourable 
effects are shown by saturated fats, mainly of animal origin. However, the results of the recently published reports indicate also the unfavourable effects of oxidised vegetable oils on the liver, as well as on the vascular walls by causing the onset of atherosclerotic lesions in the arteries. It exerts a negative influence on the parameters of lipid metabolism and oxidative stress. Such a diet is responsible for elevated concentration of total cholesterol, LDL cholesterol, triacylglycerols (TG), as well as reduction of HDL cholesterol concentration in blood, increase in concentration of malondialdehyde in organs, and reduction of antioxidative defences (5). They exert chemotactic influence on $\mathrm{T}$ lymphocytes and monocytes, intensify thrombotic processes, mainly by influencing the aggregation of thrombocytes. Oxidised LDL stimulates macrophages to produce pro-inflammatory substances, free radicals, and growth factors and is cytotoxic towards cells of the vascular walls. Incoming macrophages phagocyte lipoproteins and subsequently undergo decomposition. The decomposition of macrophages, in turn, is the source of proteinases and oxygen free radicals, which intensify lesions in vascular walls. By activation of the inflammatory process, oxidised lipoproteins may contribute to all stages of atherogenic process (18).

Application of high fat diet, as well as consumption of lipids in oxidised form induces numerous dangerous changes in the liver. High fat diet is one of the causes of non-alcoholic steatohepatitis (NASH) - considered to be the most chronic disease of this organ. Accumulation of fats in hepatocytes (steatosis of liver cells) is connected with disturbed balance between the amount of fatty acids which reach the liver, and oxidation and synthesis of triglycerides (TG), as well as release of VLDL lipoproteins. The process of TG synthesis is much faster than the process of capturing apolipoproteins from blood and TG bonding to them, which results in TG accumulation in hepatocytes. Steatosis may also be a result of metabolic inhibition of the lipoprotein generation in plasma (protein deficit in diet), due to which storage of TG in the liver takes place $(3,15,22,24)$.

Additionally, it was demonstrated that oxidative stress generated in the liver due to excessive oxidation of fatty acids may be the factor which initiates the cascade of events leading to NASH (7).

The above data confirm the oldest and commonly known hypothesis of NASH pathogenesis, the two-hit hypothesis put forward in 1998 by Day and James. According to them, the first hit consists of excessive production of triglycerides in hepatocytes, whereas in the second hit oxidative stress contributes to steatosis turning into inflammation (16).

As experimental studies have shown, one of the methods of counteracting lesions resulting from exposure to oxidised lipids is the strengthening of body antioxidant system by supplementing the diet with vitamin and non-vitamin antioxidants, which is a part of the defence system against free radicals $(20,21)$.

The aim of this study was to assess the influence of $\alpha$-lipoic acid (ALA) on the morphology of the liver and aorta in rabbits kept on high fat diet with the addition of fresh or oxidised rapeseed oil.

ALA and its reduced form-dihydroliponic acid are the ideal antioxidants because they can easily scavenge free radicals, removing hydroxyl radicals, peroxynitrite radicals $\left(\mathrm{NOO}^{-}\right)$, and oxygen radicals. They can chelate metals, have an amphiphilic properties (they are able to function both in the aqueous phase as well as in the lipid phase), and do not exhibit any serious side effects when used. They can also interact with other antioxidants and can regenerate them. All of those features make them one of the most universal antioxidants available $(13,29)$.

\section{Material and Methods}

The study was conducted on 36 Chinchilla rabbits of initial body weight of 2,800 $\pm 200 \mathrm{~g}$ and about three months of age. The animals were bred in the Centre of Experimental Medicine of the Medical University of Silesia in Katowice, Poland.

The experiment lasted six months and was preceded by a two-week adaptation of animals to experimental conditions.

Throughout the study, each rabbit was kept in a stainless steel separate metabolic cage under the day/night cycle. The animals received water ad libitum.

The rabbits were divided into six groups of six rabbits in each group. Control group (C) - fed a breeding standard diet (BSD), group I (BSD+ALA) received ALA in the dose of $10 \mathrm{mg} / \mathrm{kg} \mathrm{b.w.,} \mathrm{group} \mathrm{II}$ (BSD+N-ORO) received $10 \%$ addition of no-oxidised rapeseed oil (N-ORO), group III (BSD+ORO) received $10 \%$ addition of oxidised rapeseed oil (ORO), group IV $(\mathrm{BSD}+\mathrm{N}-\mathrm{ORO}+\mathrm{ALA})$ received $10 \%$ addition of $\mathrm{N}-\mathrm{ORO}$ and ALA in the dose of $10 \mathrm{mg} / \mathrm{kg} \mathrm{b.w.,} \mathrm{and}$ group $\mathrm{V}(\mathrm{BSD}+\mathrm{ORO}+\mathrm{ALA})$ received $10 \%$ addition of ORO and ALA in the dose of $10 \mathrm{mg} / \mathrm{kg} \mathrm{b.w}$. The daily dose was $80 \mathrm{~g}$ of fodder $/ \mathrm{kg}$ of rabbit body weight. With an increase in body weight, increasing amount of fodder was provided, while the concentration of ingredients remained unchanged.

Addition of $10 \%$ of rapeseed oil to standard diet caused the change of diet to a high fat diet ( $42 \%$ of fat). Oil oxidation was performed in accordance with standardisation parameters. It was conducted for $6 \mathrm{~h}$ at $180^{\circ} \mathrm{C}$ in glass vessels of $40 \mathrm{~cm}$ in diameter (volume of $1 \mathrm{~L}$ in the vessel) in a laboratory drier with forced circulation. The daily dose of oil was $8 \mathrm{~g} / \mathrm{kg}$ of rabbit body weight. Additional ingredients were added at the feed preparation stage, before the fodder was finally granulated and prepared in portions sufficient for one month of feeding. 
The analysis of fodder consumption was conducted by assessing the food residues left. The content of breeding standard diet (BSD) per $100 \mathrm{~g}$ of fodder was: $13 \mathrm{~g}$ of protein, $2.9 \mathrm{~g}$ of fats, $11 \mathrm{~g}$ of fibre, and $49 \mathrm{~g}$ of carbohydrates $(24 \%$ energy came from protein, $7 \%$ from fats, $69 \%$ from carbohydrates). Ten percent addition of rapeseed oil caused the diet to change into high fat diet: $18 \%$ of energy came from protein, $42 \%$ from fats, and $39 \%$ from carbohydrates.

After six months the animals were killed by exsanguinations from the carotid artery, under deep general anaesthesia, achieved by administration of a mixture of ketamine, droperidol, and fentanyl (doses of $50 \mathrm{mg} / \mathrm{kg}$ b.w., $0.1 \mathrm{mg} / \mathrm{kg}$ b.w., and $0.1 \mathrm{mg} / \mathrm{kg} \mathrm{b.w.,}$ respectively). Specimens of the organs were collected for pathomorphological assessment.

Histopathological evaluation. The collected organs were fixed in formalin. Pathomorphological lesions were assessed on paraffin sections. The sections were stained with haematoxylin and eosin (H-E). For detection of neutral fats, the preparations were made using freezing method and stained with Sudan III (33). The preparations were examined under different magnifications $(100 \times, 200 \times, 400 \times$, and $600 \times)$ under light Olympus microscope. Microphotographs were taken using the Olympus Camedia C-3040 camera.

\section{Results}

Macroscopic and microscopic assessment of the liver. Macroscopic lesions were detected only in the liver of the rabbits which received oxidised rapeseed oil (group III). The liver of all animals from this group had a lighter tint and were slightly yellowish in comparison with the liver from control and other groups.

The results of pathomorphological assessment of preparations are shown in 10 figures. In the liver from control group $(\mathrm{C})$ no changes were found (Figs 1A, 1B).

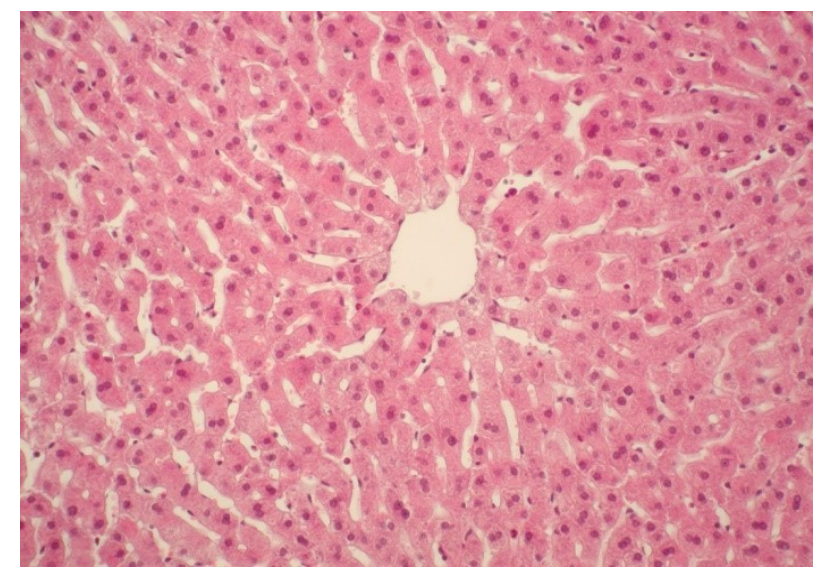

Fig. 1A. Liver. Control group (BDS). No lesions in hepatocytes or liver stroma. H-E, $200 \times$

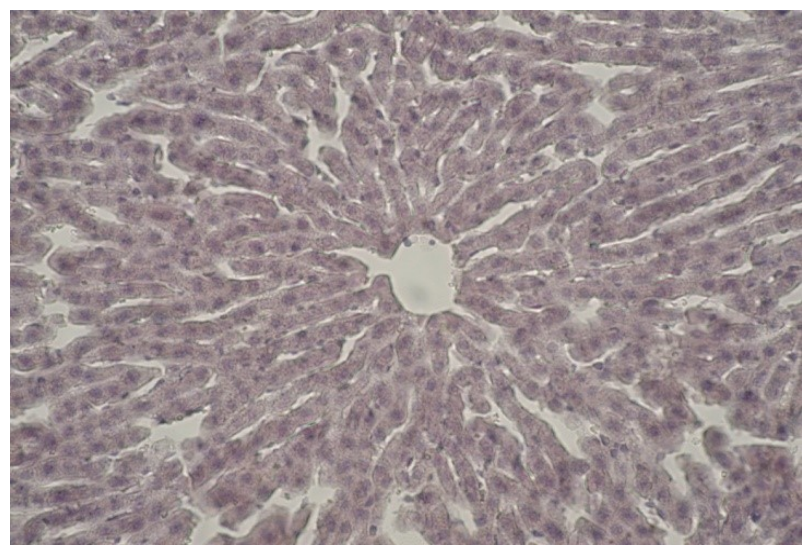

Fig. 1B. Liver. Control group (BDS). No steatosis in hepatocytes. Sudan III, 200×

In group I (BSD+ALA), slight infiltrations with mononuclear cells and intensified activity of BrowiczKupffer cells were observed in two rabbits (Fig. 2A). In the liver from group II (BSD+N-ORO) infiltrations of mononuclear cells were observed in the area of liver triads and between liver lobules (Fig. 3A). No fatty degeneration in hepatocytes was noted.

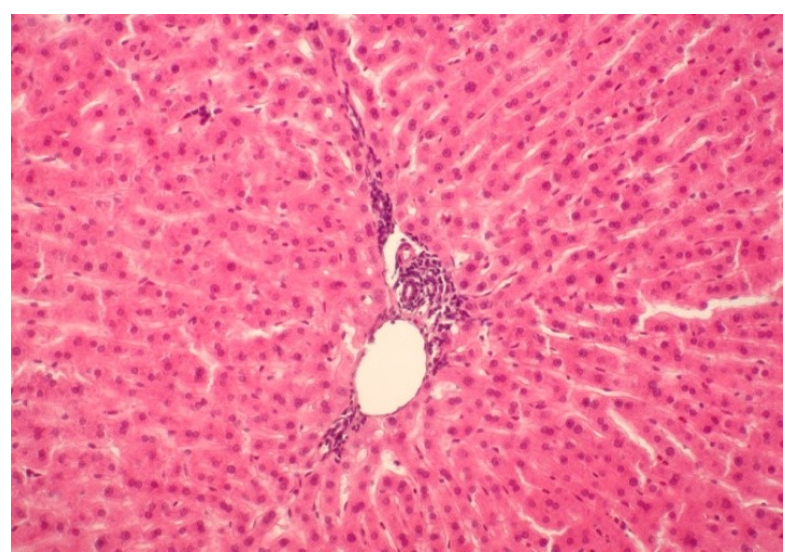

Fig. 2A. Liver. Group I (BDS+ALA). No lesions in hepatocytes. Slight infiltrations with mononuclear cells in the liver triad. H-E, $200 \times$

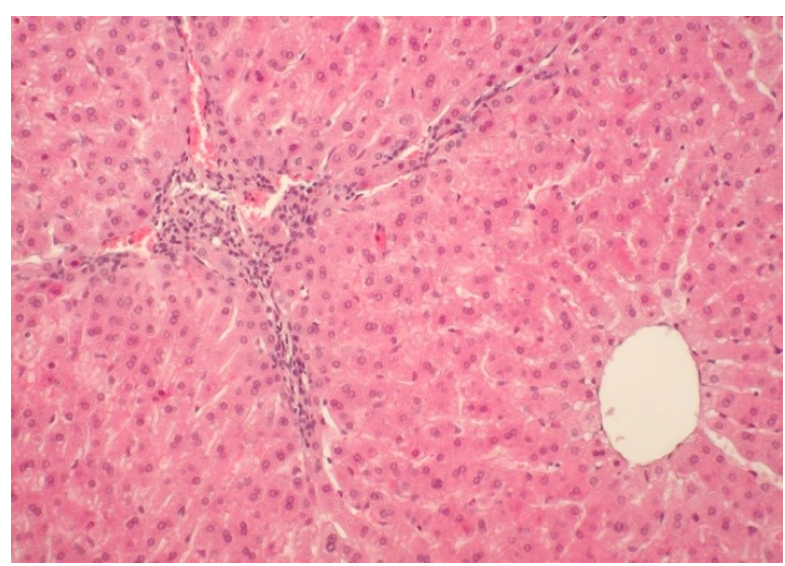

Fig. 3A. Liver. Group II (BSD+N-ORO). Mononuclear cell infiltrations in the liver triad area and between liver lobules. No lesions in hepatocytes. H-E, 200× 
The most intensified pathomorphological changes were found in animals from group III (BSD+ORO). Retrogressive changes in the form of focal necrosis of hepatocytes and steatosis of liver cells were observed in two rabbits (Figs 4A, 4B). The administration of ALA to rabbits fed a diet containing N-ORO (group IV) and ORO (group V) demonstrated beneficial influence of ALA in counteracting the lesions noted in the liver after exposure to the oils. The full effectiveness of ALA in group IV was demonstrated (Figs 5A, 5B). In group $\mathrm{V}$ no necrotic lesions were observed in the liver cells, yet in case of two animals the presence of infiltrations with mononuclear cells in the liver triad area (Fig. 6A) and small steatosis of hepatocytes around the central vein (Fig. 6B) were observed.

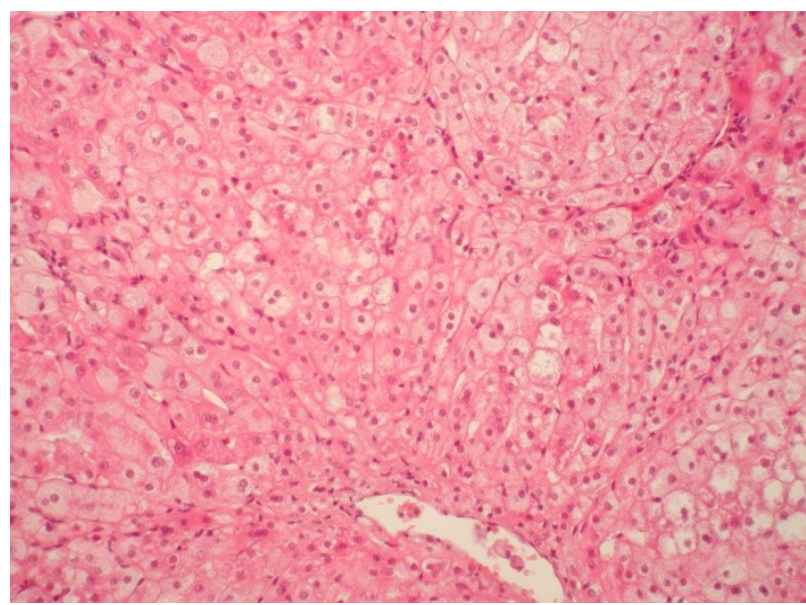

Fig. 4A. Liver. Group III (BSD+ORO). Focal necrosis and steatosis of hepatocytes. H-E, 400×

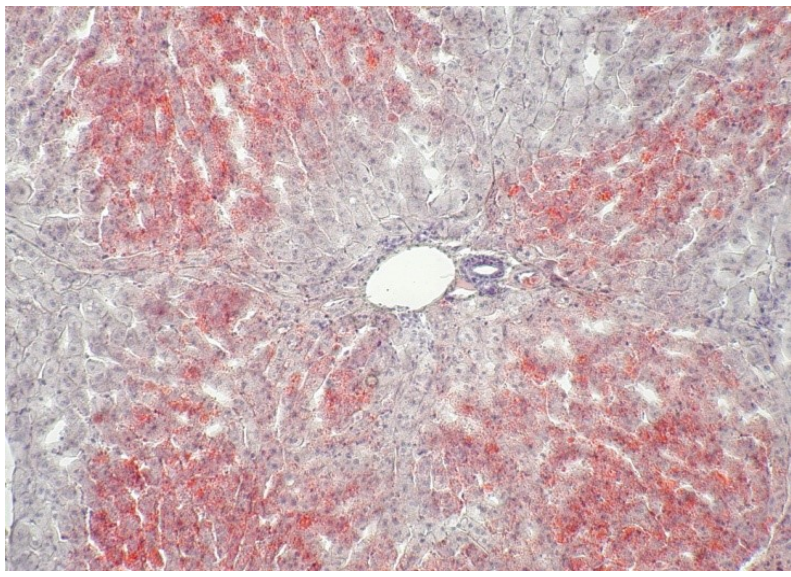

Fig. 4B. Liver. Group III (BSD+ORO). Intense steatosis in liver lobule. Sudan III, 200×

Macroscopic and microscopic assessment of aorta. In the aortas collected from four rabbits from Group III (BSD+ORO) focal or complete peripheral thickening of vessel wall was observed (internal layer hyperplasia). No macroscopic lesions in aortas were observed in other groups.

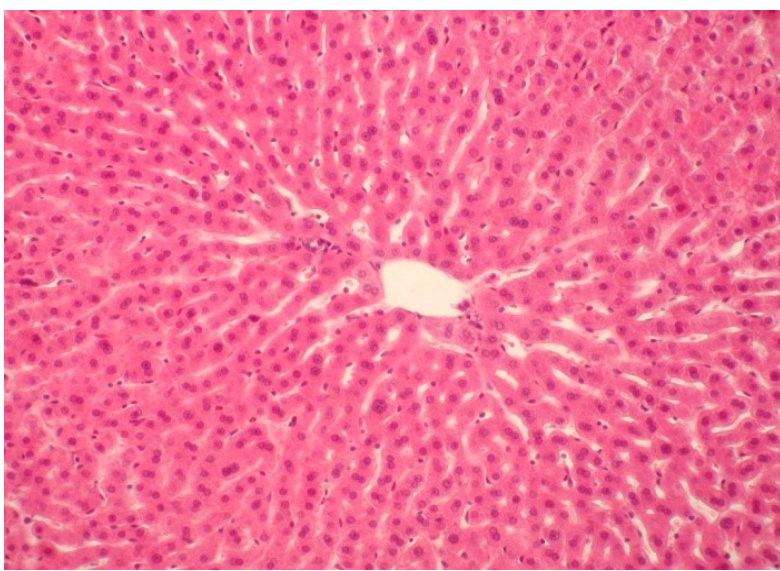

Fig. 5A. Liver. Group IV (BSD+N-ORO+ALA). No lesions in hepatocytes. H-E, 200×

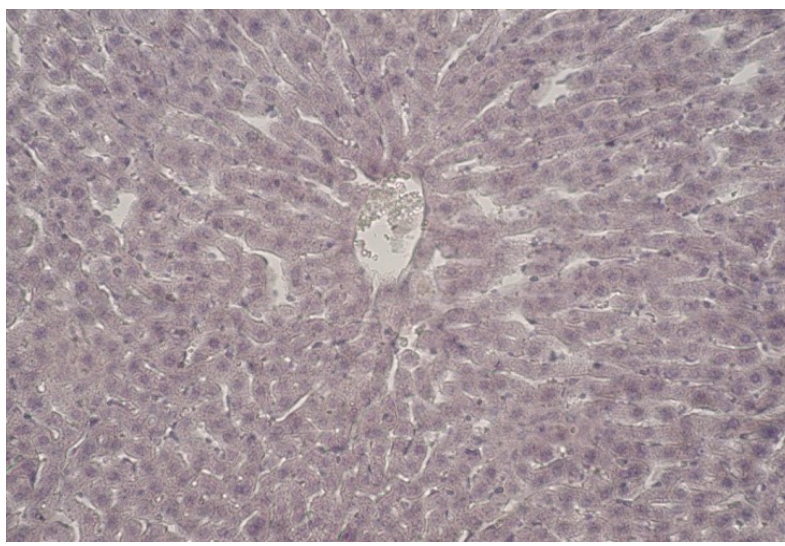

Fig. 5B. Liver. Group IV (BSD+N-ORO+ALA). Normal image. No steatosis in liver cells. Sudan III, 200×

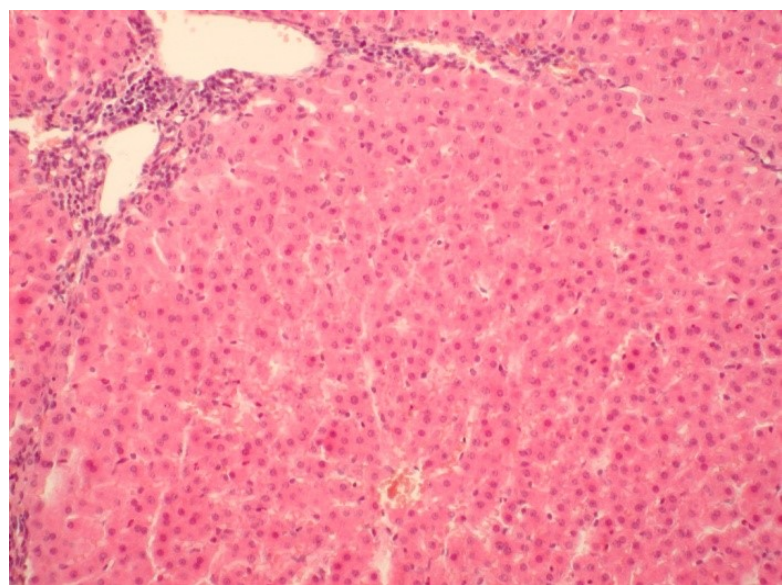

Fig. 6A. Liver. Group V (BSD+ORO+ALA) Abundant infiltration of mononuclear cells in the liver triad area and between hepatocytes. H-E, 200×

In group I (BSD+ALA) no microscopic changes were seen in the aortas (Fig. 8A). In group II (BSD+NORO) slight oedema of the internal layer (elastic lamina diastasis) was observed in the aortas (Fig. 9A). 


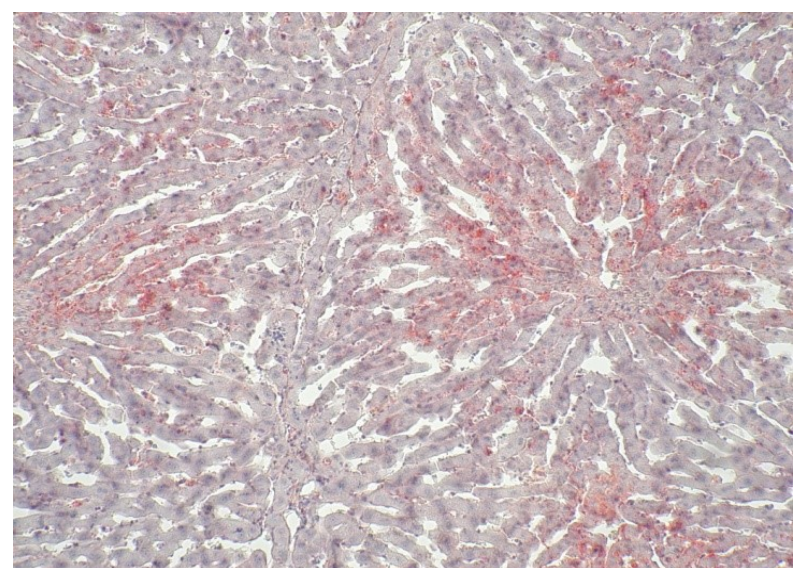

Fig. 6B. Liver. Group V (BSD+ORO+ALA). Small steatosis of hepatocytes around the central vein. Sudan III, 200×

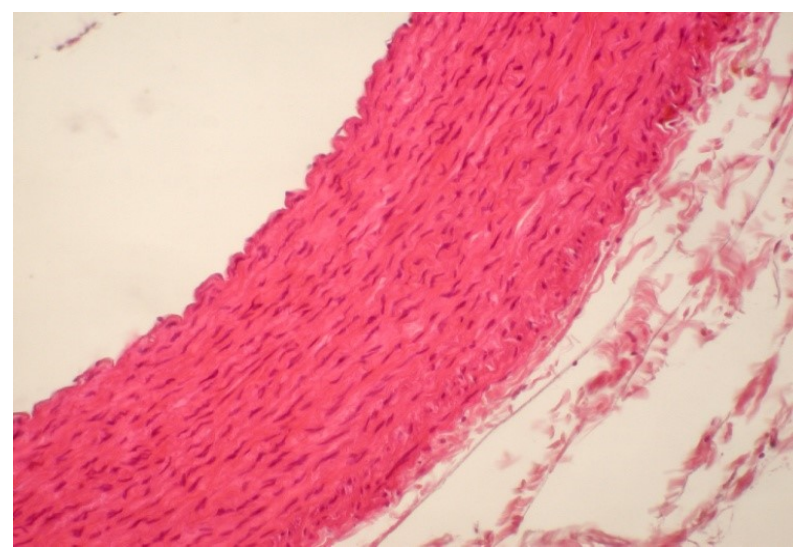

Fig. 7A. Aorta. Control group (BDS). Normal pattern. H-E, 200×

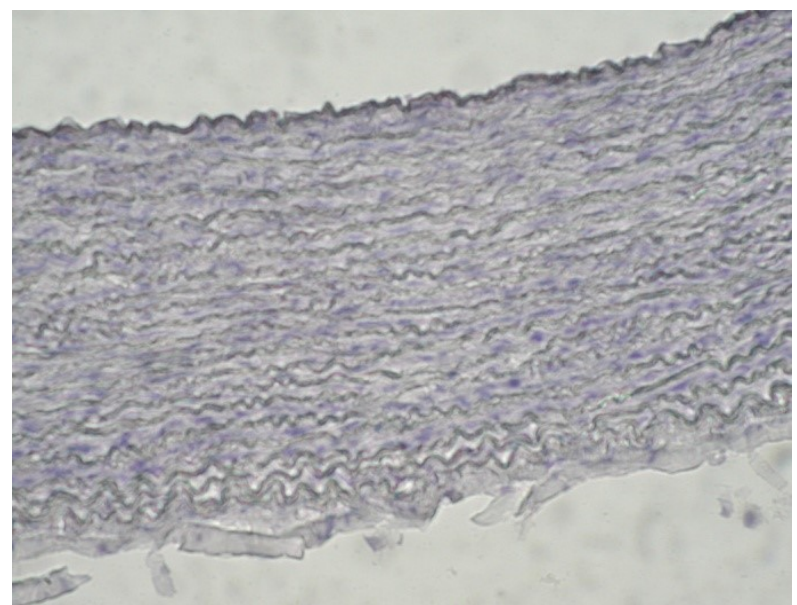

Fig. 7B. Aorta. Control group (BDS). No lesions in aorta wall. Sudan III, 200×

In group III (BSD+ORO), the presence of a substantial atherosclerotic plaque was detected in the aorta of two rabbits. The plaque revealed significant fatty infiltrations and foam cells. Numerous myocytes, lymphocytes, and fibres were present in the area. Lesions of elastic fibres and the presence of macrophages were also observed. The aorta wall was significantly thicker and its lumen was narrowed (Figs 10C, 10D). The lesions noted in two other rabbits were not so profound (Figs 10A, 10B).

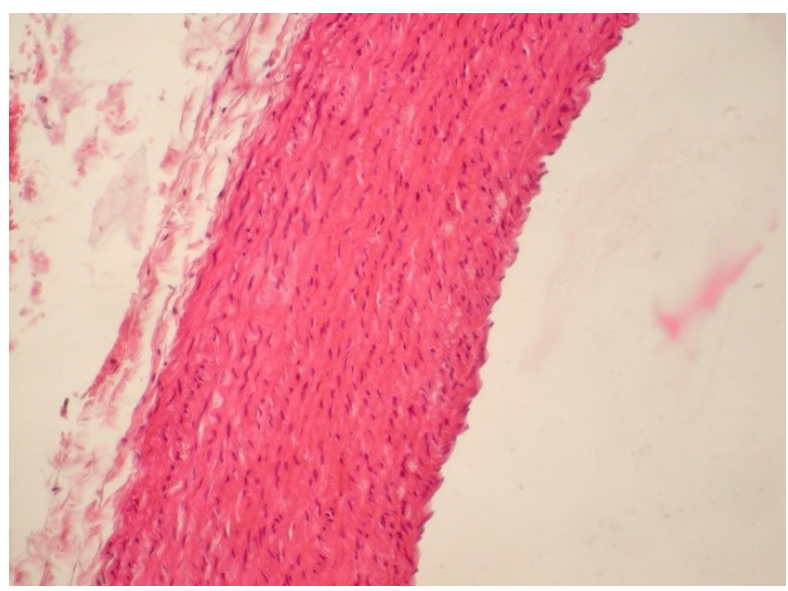

Fig. 8A. Aorta. Group I (BDS+ALA). No lesions in aorta wall. H-E, $200 \times$

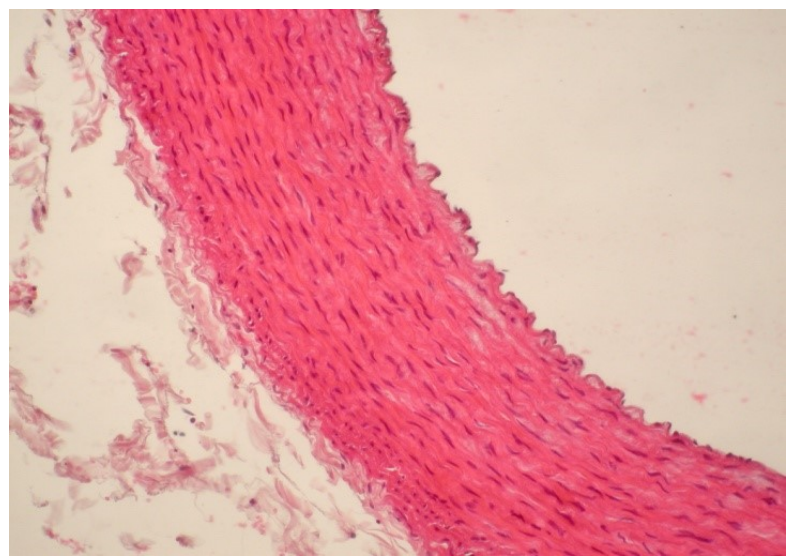

Fig. 9A. Aorta. Group II (BSD+N-ORO). No lesions. H-E, 200×

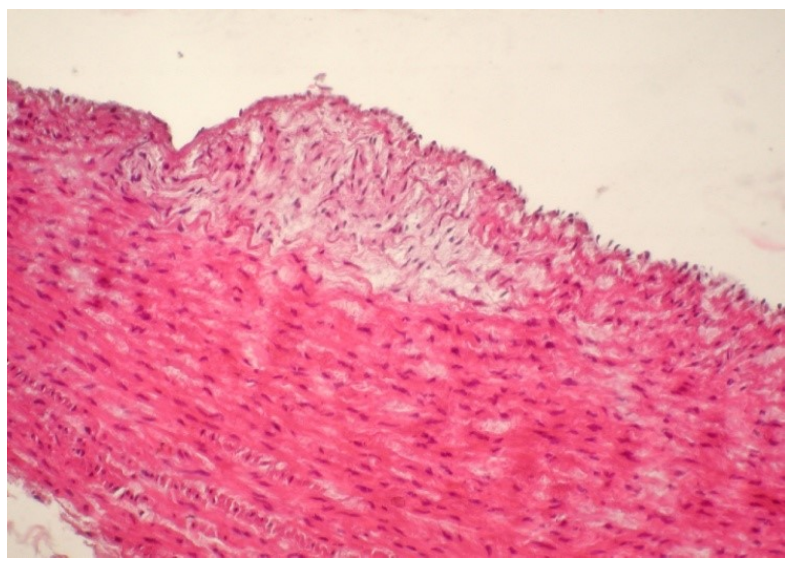

Fig. 10A. Aorta. Group III (BSD+ORO). Small atherosclerotic plaque in which foam cells are visible. H-E, 200× 


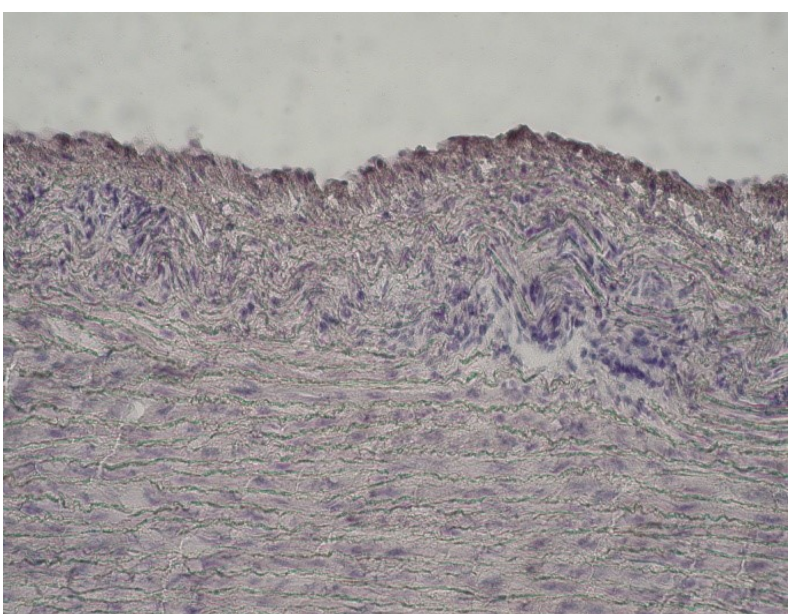

Fig. 10B. Aorta. Group III (BSD+ORO). Hyperplasia of internal layer. Numerous oocytes and macrophages in internal layer. Sudan III, 200×

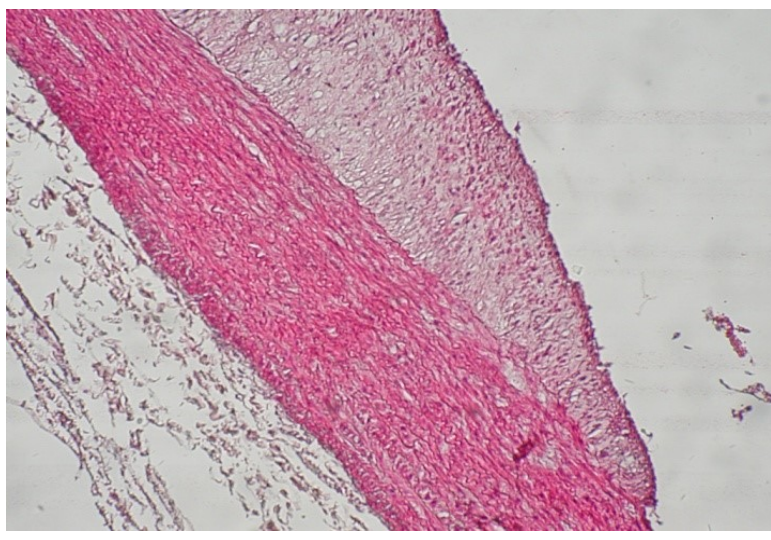

Fig. 10C. Aorta. Group III (BSD+ORO). Large atherosclerotic plaque. In the plaque foam cells, extracellular fat, smooth myocytes, macrophages, and fibrosis can be seen. Internal elastic lamina is not visible. H-E, $100 \times$

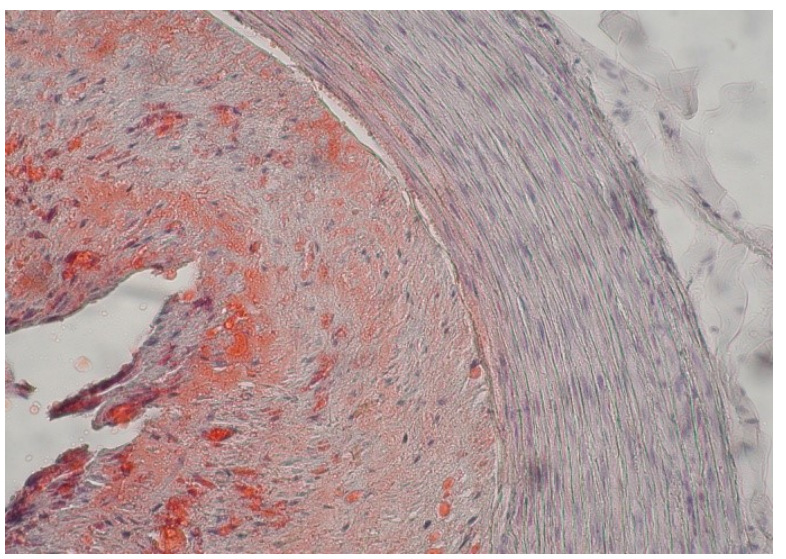

Fig. 10D. Aorta. Group IV (BSD+ORO). Large atherosclerotic plaque with foam cells and extracellular fat, stained in orange, smooth myocytes, macrophages, and fibrosis. Internal elastic lamina is not visible. Sudan III, 100×

In rabbits from group IV (BSD+N-ORO+ALA) no changes in the aorta walls were observed (Figs 11A,
11B), whereas in two rabbits from group $\mathrm{V}$ (BSD+ORO+ALA) only slight focal proliferation of myocytes and atherosclerotic plaque were noted (Figs $12 \mathrm{~A}, 12 \mathrm{~B})$.

The structure of aorta wall in controls was not changed (Figs 7A, 7B).

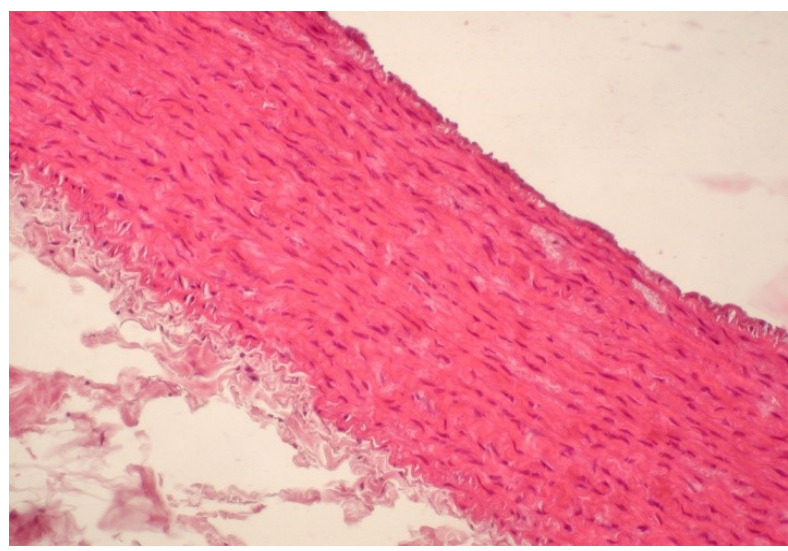

Fig. 11A. Group IV (BSD+N-ORO+ALA). No lesions in aorta wall. H-E, 200×

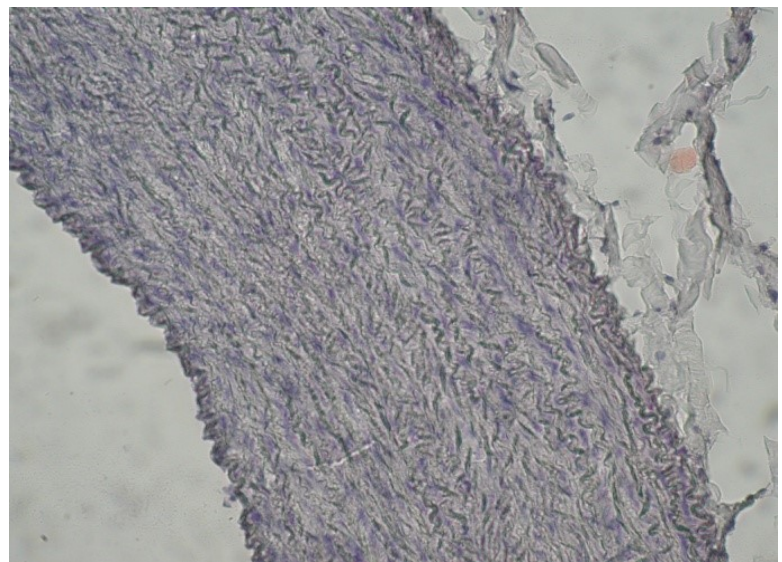

Fig. 11B. Aorta. Group IV (BSD+N-ORO+ALA). No lesions in aorta wall. Sudan III, $200 \times$

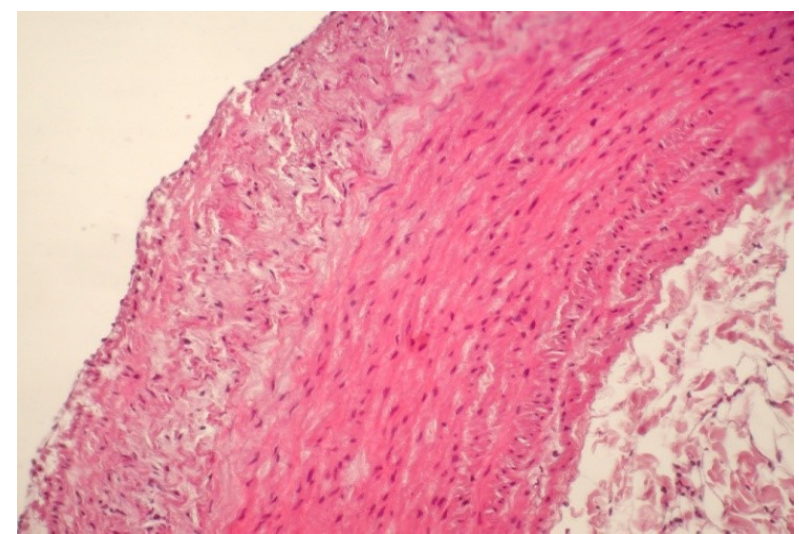

Fig. 12A. Aorta. Group V (BSD+ORO+ALA). Atherosclerotic plaque with foam cells and macrophages. H-E, 200× 


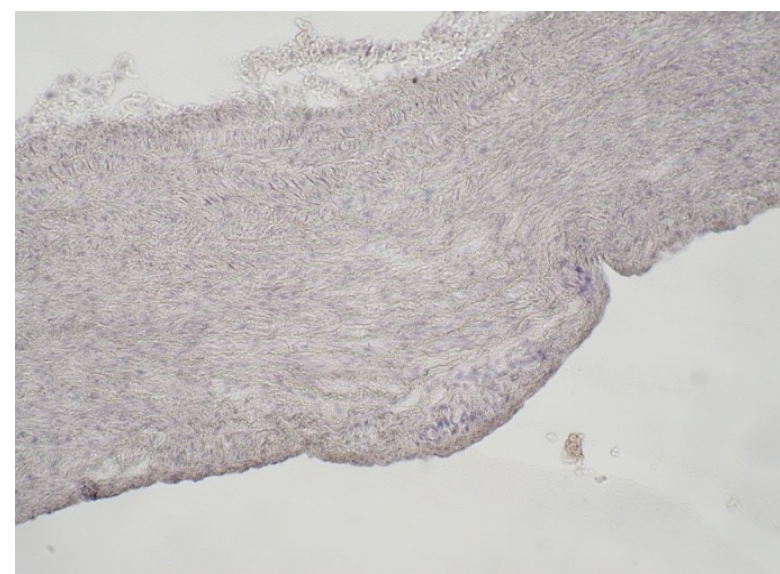

Fig. 12B. Aorta. Group V (BSD+ORO+ALA. Small atherosclerotic plaque with macrophages. No fats in the plaque. Sudan III, 200×

\section{Discussion}

The excess or deficiency of foodstuffs, as well as their inadequate quality results in specific consequences for health (6). Excess of fats and oxidative transformations of fats is a particularly important problem, as they lead to deterioration of the quality of food products and reduction of their nutritional and biological value. Due to oxidation fats become a rich source of free radicals, which are a threat to health. They undergo oxidation mainly because of frying and prolonged and improper storage. Deep frying causes hydrolysis, oxidation, and polymerisation of oil. The products of fat oxidation include mainly hydroxides and volatile compounds with low molecular weight, such as aldehydes, ketones, alkanes, and carboxyl acids. These compounds play a vital role in intensification of oxidative stress $(4,10,26)$.

Recently, intensive research has been conducted to provide a detailed explanation of the influence of high fat diet and the quality of consumed fats upon the development of cardio-vascular diseases. It has been demonstrated that a diet containing oxidised lipids is one of the main risk factors of coronary diseases and atherosclerosis of the aorta or renal arteries. It exerts a negative influence on the parameters of lipid metabolism, as well as oxidative stress. Such a diet is responsible for elevated concentration of triacylglyceroles, total cholesterol, cholesterol of the LDL fraction, and reduction of HDL cholesterol concentration in blood, increase in concentration of malondialdehyde in organs, and reduction of antioxidative defence (5).

High fat diet and consumption of oxidised fats cause non-alcoholic steatohepatitis (NASH). It is assumed that accumulation of lipids and oxidative stress are the main causes of the disease. NASH may lead to cirrhosis $(19,26)$.

It has been demonstrated that impaired functions and mitochondrial structures in hepatocytes contribute to the development of liver steatosis. In mitochondria isolated from rats fed a high fat diet, reduced rate of mitochondrial oxidation and increased rate of reactive oxygen species production was observed (16).

Our study revealed morphological changes in the liver and aorta of animals fed the BSD+ORO diet. The addition of ORO caused focal and dispersed necrosis of hepatocytes and steatosis of liver cells. The results indicate that oxidised fats in diet may significantly damage the liver, limiting its functions. On the other hand, slight changes were observed in the liver of animals which received $\mathrm{BSD}+\mathrm{N}-\mathrm{ORO}$ fodder. Only slight infiltrations of mononuclear cells were observed in the liver triad area, as well as between liver lobules, which occurred in two animals. This may be the answer to excessive inflow of fat to the liver.

The results obtained in our study correspond with the results of Meli et al. (14) who demonstrated that feeding rats a diet rich in fat leads to a gradual increase in steatosis and the emergence of infiltrations of inflammatory cells in the liver and necrosis of hepatocytes. The authors also noted increased concentration of lipid parameters and liver indicator enzymes (ALT and AST) in blood serum. Moreover, studies conducted on rats showed that high fat diet inhibited synthesis of apoproteins and lipoproteins, and disturbed the transport of fatty acids to cells in various tissues (34). Totani et al. (23), demonstrated steatosis and necrotic lesions in the liver and kidneys of rats fed a diet with addition of fried oil. Similar experimental studies on rabbits revealed that a diet containing ORO contributed to the development of slight steatosis of the liver and increased activity of enzymes participating in liver metabolic pathways, such as AST, ALT, GLDH, and SDH (30).

Our study demonstrated also that addition of ORO to diet resulted in the development of different intensity atherosclerotic plaque in four animals. Fatty infiltrations, foam cells, numerous myocytes, macrophages, lymphocytes, and fibrosis were clearly visible in the plaque, and the aortic media were involved in the process in two rabbits. In other two rabbits the lesions of lower intensity were also found. On the other hand, supplementation of diet with $\mathrm{N}-\mathrm{ORO}$ led only to the oedema of aorta internal layer in one rabbit.

The results of pathomorphological assessment presented here confirmed the influence of oxidised lipids on atherogenesis, shown earlier in numerous studies related to the assessment of the relation between high fat diet and oxidised fat and lipid levels in blood serum and risk of atherosclerosis development in animal models $(2,16,21,30)$. The high concentration of lipoproteins with low density of LDL is a well recognised risk factor for development of coronary heart diseases. Oxidative modification of LDL leads to increased LDL capture by macrophages in the artery wall. Thus, formed foam cells are the basic component 
of atherosclerotic lesions in vascular wall (18). Similar effects were noted in the studies on hamsters (8) and rabbits (12) fed a high fat diet. Lesions in aorta internal layer were also demonstrated by Wang et al. (25). The results of our study confirmed the negative influence of oxidised oil on the aorta.

A properly composed diet plays a very important role in prevention of diseases of the liver and circulatory system. Reducing the amount of fat consumed, as well as paying attention to its quality, is crucial to reduce the risk of these diseases. It is also important to include in a diet products containing natural antioxidants, which can protect the organism from various unfavourable changes, despite consumption of oxidised lipids.

This study attempted to assess the influence of diet supplementation with $\alpha$-lipoic acid on the morphology of rabbit organs exposed to diet containing oxidised fats. Studies conducted so far indicate that ALA, as a universal antioxidant (active both in aqueous and lipid phase), demonstrates preventive activity in relation to many diseases, the causes of which are oxido-reductive processes or, in case of pathogenesis, processes in which oxidative stress occurs $(9,11,13$, 17, 29, 31, 32).

In the present study the beneficial influence of ALA was demonstrated in relation to lesions observed in rabbits fed a diet containing N-ORO or ORO. In the case of diet containing ORO and supplemented with ALA, the activity of the acid was not fully effective. Pathomorphological lesions appeared in the aorta of two rabbits (small atherosclerotic plaque) and slight infiltrations of inflammatory cells occurred in the liver triad area in two animals. No necrotic lesions or steatosis of hepatocytes were observed. In the case of administration of ALA with N-ORO, the full effectiveness of ALA in protecting the liver and aorta was demonstrated. Beneficial effect of ALA on the hepatic lesions was previously demonstrated in rats exposed to high fat diet (60\% of fats) (11). On the other hand, Zalejska-Fiolka et al. (31) found the positive influence of ALA on changes in the parameters of oxidative system and morphological structure of the liver in rabbits exposed to oxidised olive oil. In the experimental studies, preventive properties of other antioxidants, like methionine, and selenomethionine, were also shown with reference to changes observed in animals fed a diet containing cholesterol. Less marked steatosis in the liver and small atherosclerotic plaques in aortas, coronary arteries, and renal arteries were observed $(20,21)$. On the other hand, Haliga et al. (8) demonstrated that addition of flax seed and vitamin $\mathrm{E}$ in hamsters fed high-fat diet prevented the occurrence of atherosclerotic changes within the aorta. In studies conducted on rats receiving high-fat diet, $\mathrm{Xu}$ et al. (28) demonstrated that enriching rapeseed oil with vitamin E, polyphenols, and phytosterols counteracts steatosis of the liver in the animals.
In summary, the obtained results of pathomorphological assessment of the liver and aorta indicate the risk of atherosclerosis and steatosis of the liver in case of exposure to oxidised fats in diet. Diet supplementation with $\alpha$-lipoic acid counteracted the changes generated in these organs under increased exposure to higher fat content, in particular thermally treated fats, in diet.

Conflict of Interests Statement: The authors declare that there is no conflict of interests regarding the publication of this article.

Financial Disclosure Statement: The study was supported by project of the Medical University of Silesia in Katowice Nos. KNW-1-031/N/4/0, KNW-1-017/08, KNW-1-051/09, KNW-1-046/10.

Animal Rights Statement: The experiment was approved by the Ethical Committee of the Medical University of Silesia in Katowice, Poland.

\section{References}

1. Berliner J.A., Heinecke W.J.: The role of oxidized lipoproteins in atherogenesis. Free Radic Biol Med 1996, 20, 707-727.

2. Brukow K.G.: Oxidized lipids: The two faces of vascular inflammation. Curr Atheroscler Rep 2006, 8, 223-231.

3. Bukol-Krawczyk K.: Nonalcoholic steatosis of the liver - to treat or not to treat? Forum Metabol Disord 2010, 1, 66-72.

4. Choe E., Min D.B.: Chemistry of deep-fat frying oils. J Food Sci 2007, 72, 77-86.

5. Cohn J.: Oxidized fat in the diet, postprandial lipaemia and cardiovascular disease. Curr Opin Lipidol 2003, 13, 19-24.

6. Fiedurek J.: The role of food and nutrition in prophylaxis and therapy of human diseases. Edition of University of Maria CurieSklodowska, Lublin, 2007, pp. 46-47.

7. Garnol T., Endlicher R., Kucera O., Drahota Z., Cervinkova Z.: Impairment of mitochondrial function of rat hepatocytes by high fat diet and oxidative stress. Physiol Res 2014, 63, 271-274.

8. Haliga R.E., Mocanu V., Badescu M.: Antioxidative and antiatherogenic effects of flaxseed, $\alpha$-tocopherol and their combination in diabetic hamsters fed with a high-fat diet. Exp Ther Med 2015, 9, 533-538.

9. Ide T., Azechi A., Suzuki N., Kunimatsu Y., Nakajima C., Kitade S.: Effects of dietary a-lipoic acid enantiomers on hepatic fatty acid metabolism in rats. J Funct Foods 2013, 5, 71-79.

10. Kanner J.: Dietary advanced lipid oxidation endproducts are risk factors to human health Mol Nutr Food Res 2007, 51, 1094-1101.

11. Kaya-Dagistanlia F., Tanriverdi G., Altinok A., Ozyazgan S., Ozturk M.: The effects of alpha lipoic acid on liver cells damages and apoptosis induced by polyunsaturated fatty acids. Food Chem Toxicol 2013, 53, 84-93.

12. Lai P., Du J., Zhang M., Kuang X., Li Y.J., Chen Y.S., He Y.: Aqueous extract of Gleditsia sinensis Lam. fruits improves serum and liver lipid profiles and attenuates atherosclerosis in rabbits fed a high-fat diet. J Ethnopharmacol 2011, 137, 1061-1066.

13. Malińska D., Winiarska K.: Lipoic acid: characteristics and therapeutic application. Post Hig Med Dosw 2005, 59, 535-543.

14. Meli R., Mattace Raso G., Irace C., Simeoli R., Di Pascale A., Paciello O., Pagano TB., Calignano A., Colonna A., Santamaria R.: High fat diet induces liver steatosis and early dysregulation of 
iron metabolism in rats. Plos One 2013, 8, e66570, doi: 10.1371/journal.pone.0066570.

15. Neuschwander-Tetri B.A.: Hepatic lipotoxicity and the pathogenesis of nonalcoholic steatohepatitis: the central role of nontriglyceride fatty acid metabolites. Hepatology 2010, 52, 774-788

16. Noeman S., Hamooda H., Baalash A.: Biochemical study of oxidative stress markers in the liver, kidney, and heart of high fat diet induced obesity in rats. Diabetol Metab Syndr 2011, doi: 10.1186/1758-5996-3-17.

17. Petersen Shay K., Moreau R.F., Smith E.J., Smith A.R., Hagen T.M.: Alpha-lipoic acid as a dietary supplement: Molecular mechanisms and therapeutic potential. Biochim Biophys Acta 2009, 1790, 1149-1160.

18. Skoczyńska A.: The role of lipids in atherogenesis. Postepy Hig Med Dosw 2005, 59, 346-357.

19. Staprans I., Rapp J.H., Pan X.M, Feingold K.R.: Oxidized lipids in the diet are incorporated by the liver into low density lipoprotein in rats. J Lipid Res 1996, 37, 420-430.

20. Stawiarska-Pięta B., Birkner E., Szaflarska-Stojko E., Stojko R., Zalejska-Fiolka J., Kasperczyk S., Birkner E.: Influence of selenomethionine on the morphology of rabbits' organs in experimental atherosclerosis. Bull Vet Inst Pulawy 2006, 50, 113-119.

21. Stawiarska-Pięta B., Birkner E., Szaflarska-Stojko E., Stojko R., Grucka-Mamcza E., Pyrsak M., Wyszyńska M.: The influence of diet supplementation with methionine on the pathomorphological changes of rabbit organs in experimental atherosclerosis. Arch Med Sci 2008, 4, 371-379.

22. Totani N., Burenjargal M., Yawata M., Ojiri Y.: Chemical properties and cytotoxicity of thermally oxidized oil. J Oleo Sci 2008, 57, 153-160.

23. Totani N., Ojiri Y.: Mild ingestion of used frying oil damages hepatic and renal cells in Wistar rats. J Oleo Sci 2007, 56, 261-267.

24. Trauner M., Arrese M., Wagner M.: Fatty liver and lipotoxicity. Biochim Biophys Acta 2010, 1801, 299-310.

25. Wang S., Gao X., Li P., Liu S., Zeng Y.: Chronic unpredictable mild stress combined with a high-fat diets aggravates atherosclerosis in rats. Lipids Health Dis 2014, 13, 77. doi: 10.1186/1476-511X-13-77.

26. Wąsowicz E., Gramza A., Hęś M., Jeleń H.H., Korczak J., Małecka M., Mildner -Szkudlarz S., Rudzińska M., Samotyja U., Zawirska- Wojtasiak R.: Oxidation of lipids in food. Pol J Food Nutr Sci 2004, 13/54, 87-100.

27. Wisniewski J.R., Friedrich A., Keller T., Mann M., Koepsell H.: The impact of high-fat diet on metabolism and immune defense in small intestine mucosa. J Proteome Res 2015, 14, 353-365.

28. Xu J., Zhou X., Gao H., Chang Ch., Deng Q., Huang Q., Ma J., Wan Z., Yang J., Huang F.: Micronutrients-fortified rapeseed oil improves hepatic lipid accumulation and oxidative stress in rats fed a high-fat diet. Lipids Health Dis 2013, 12, 28.

29. Yang R., Li W., Shi Y., Le G.: Lipoic acid prevents high-fat dietinduced dyslipidemia and oxidative stress: A microarray analysis. Nutrition 2008, 24, 582-588.

30. Zalejska-Fiolka J., Kasperczyk O., Kasperczyk S., StawiarskaPięta B., Fiolka R., Birkner E. Influence of non-oxidised and oxidised rapeseed oil consumption on liver metabolism pathways and non-alcoholic steatohepatitis development in rabbits. Bull Vet Inst Pulawy 2012, 56, 255-259.

31. Zalejska-Fiolka J., Wielkoszynski T., Rokicki W., Dąbrowska N., Strzelczyk J.K., Kasperczyk A., Owczarek A., Błaszczyk U., Kasperczyk S., Stawiarska-Pięta B., Birkner E., Gamian A.: The influence of $\alpha$-lipoic acid and garlic administration on biomarkers of oxidative stress and inflammation in rabbits exposed to oxidized nutrition oils. Biomed Res Int 2015, 1-11, doi: $10.1155 / 2015 / 827879$.

32. Zalejska-Fiolka J., Wielkoszyński T., Kasperczyk S., Kasperczyk A., Birkner E.: Effects of oxidized cooking oil and $\alpha$-lipoic acid on liver antioxidants: enzyme activities and lipid peroxidation in rats fed a high fat diet. Biol Trace Elem Res 2010, 138, 272-281.

33. Zawistowski S., Basis of Histological Technique, Histology and Histopathology. PZWL, Warsaw 1986, pp. 108-122.

34. Zhukova NV., Novgorodtseva T.P., Denisenko Y.K.: Effect of the prolonged high-fat diet on the fatty acid metabolism in rat blood and liver. Lipids Health Dis 2014, 13, 49, doi: 10.1186/1476-511X-13-49 